\title{
Analysis of optical Fe II emission with the X-ray properties of a selected sample of I Zw 1 type objects
}

\author{
Bynish Paul ${ }^{1,2 *}$, Hartmut Winkler ${ }^{1}$, Stephen Potter ${ }^{1,2}$ \\ ${ }^{1}$ Department of Physics, University of Johannesburg \\ P.O. Box 524, 2006 Auckland Park, Johannesburg, South Africa. \\ ${ }^{2}$ South African Astronomical Observatory (SAAO) \\ P.O. Box 9, 7935 Observatory, Cape Town, South Africa. \\ E-mail: bynish@saao.ac.za, hwinkler@uj.ac.za, sbp@saao.ac.za
}

\begin{abstract}
We report the analysis of medium resolution optical spectra of I Zwicky 1 type objects observed with the $1.9 \mathrm{~m}$ telescope at South African Astronomical Observatory (SAAO) at Sutherland. Fourteen bright objects were observed in the southern hemisphere during at least two epochs in the two year period from December 2016 to December 2018. We present our analysis of changes in line strength and profile of three objects, Mkn 1044, 1H $0707-495$ and IRAS 03450+0055. Spectra of high signal-to-noise ratio are achieved by combining between 4 and 10 spectra for each epoch. This enables us to decompose and identify the weak individual lines of Fe II and other emissions. To confirm the nature and extent of spectral changes, we compare these combined spectra between the epochs. Mkn 1044 and IRAS 03450+0055 show extended [O III]5007 blue wings with a line width of around $1000 \mathrm{~km} / \mathrm{s}$. Both [O III] blue wing and $\mathrm{H} \beta$ ILR components match the Fe II profiles, suggesting that they all originate in the same region. No dramatic flux variations are found in $\mathrm{H} \beta(<10 \%)$, Fe II bands $(<18 \%$, with majority of the Fe II bands varying less than 4\%), and in X-rays ( $<34 \%$ for $1 \mathrm{H} 0707-495$, and $<60 \%$ for Mkn 1044 between adjacent epochs). The minimum variability found in Fe II lines may imply that the origin of Fe II lines is far from the accretion disk.
\end{abstract}

7th Annual Conference on High Energy Astrophysics in Southern Africa - HEASA2019

28 - 30 August 2019

Swakopmund, Namibia

${ }^{*}$ Speaker. 


\section{Introduction}

Optical Fe II emission is one of the most interesting features of Active Galactic Nuclei (AGN) spectra. Many transitions between the energy levels of the complex Fe II ion are responsible for this emission [1]. Fe II emission can be very strong in the Narrow-Line Seyfert1 (NLS1) galaxies. When the iron-line spectrum is exceptionally prominent, these AGN are also termed as I Zwicky 1 (I Zw 1) objects. I Zw 1 objects are hence galaxies with extremely strong Fe II emission and weak forbidden lines [2,3]. Based on their optical emission line properties, the NLS1 galaxies have the following features; (i) presence of strong Fe II multiplet emissions or higher ionized Fe-lines, (ii) presence of unusually narrow permitted broad emission lines that are only slightly broader than the forbidden lines, (iii) the $[\mathrm{OIII}] / \mathrm{H} \beta$ flux ratio is less than 3 [4], and the FWHM of the $\mathrm{H} \beta$ line is smaller than about $2000 \mathrm{~km} / \mathrm{s}$ [5].

Physical conditions and geometry of emission regions may influence spectral properties of AGN such as line flux. These indicate the physical properties of the ionised AGN gas from which lines fluxes originate. The geometry and the gravity driven motion of the Broad Line Region influence the line profiles. The Doppler effect causes the line broadening due to random velocity of the gas clouds and the shifts of the lines are as a result of the systemic motions of the emitting gas clouds [1].

Many correlations have been noticed between Fe II and other emission lines, some relating to the full width at half maximum (FWHM) of $\mathrm{H} \beta$ of the observed sample $[6,7,8,9,1]$. The variability characteristic of the Fe II complex in Seyfert galaxies has not been well understood [10]. In a sample of 12 NLS1, a minimum of four AGN showed notable variability of less than $30 \%$ of the Fe II emission [11]. There is no conclusive evidence of where in an AGN the Fe II emission-line region is located. According to previous studies, the Fe II emission could originate in the intermediate line region (ILR) between the broad-line region (BLR) and the narrow-line region (NLR) [12, 13, 1], in the broad $\mathrm{H} \beta$ line region [2], in the outer part of the flattened BLR [14], or in the accretion disk surrounding the central source $[15,16]$.

The NLS1 phenomenon still poses questions related to the current AGN unified model [17]. The Fe II emission spectra formation from AGN is still not well understood $[18,10]$. The dynamics responsible for the "unusually narrow Balmer lines" in the broad line region and the unusual large Fe II strength in their spectra are still to be explained well. The source of AGN variability, the mechanisms of their excitation, and location of the Fe II emission region in AGN are still open questions [1, 19].

In this paper, we investigate the Fe II emitting region in a sample of I Zw 1 type objects by analysing the line variability, line ratios and the correlations between them within the Fe II multiplets, width and shifts of the lines between different Fe II shelves, and other prominent emission lines. Searching for a physical cause of these correlations may help in understanding the origin of Fe II emission. Using these results, we then interpret Fe II emission in terms of the current theoretical AGN model.

\section{Observations and spectral calibration}

The sample of our study consists of some of the brightest known NLS1 sources accessible from 
Sutherland. For the spectra presented here, the observations were carried out at several epochs from December 2016 to November 2018.

For all the observations, we made use of the $1.9 \mathrm{~m}$ telescope at the Sutherland Observatory, using the SAAO Optical Spectrograph (SpUpNIC). Medium resolution spectra covering a wavelength range $3500-6300 \AA$ were obtained using Grating 6 with a grating angle $12.5^{\circ}$ and a slit width of 0.9 arcsec. A Cu-Ar Lamp was used to produce an Arc spectrum for wavelength calibration purposes. We observed well-known spectrophotometric standard stars for the flux calibration.

The data reduction, including the wavelength and flux calibration, was done using standard IRAF packages. Each target presented here was exposed for 20 minute integrations and multiple such observations were carried out for each object. The spectra of the three objects presented below were obtained as a result of co-adding 4-10 spectra, which produced a high signal-to-noise ratio for each of these targets. Heliocentric and Doppler corrections were done and the continuum flux contribution was subtracted from each spectrum. To subtract the continuum the task ONEDSPEC.CONTINUUM in IRAF was used to fit a one dimensional function to the continuum. Based on the residuals between the fitted points and the fitted function, a number of iterations were done to determine the continuum. [O III]-scaling was also done to compensate for light losses caused by bad seeing or sky transparency changes during the observations. Spectral measurements of Fe II bands were performed using an Fe II model template [1]. In order to carry out spectral measurements for other prominent emission lines, Fe II lines were then subtracted from the original spectra to investigate some of the other prominent emission lines.

\section{Analysis and Discussion}

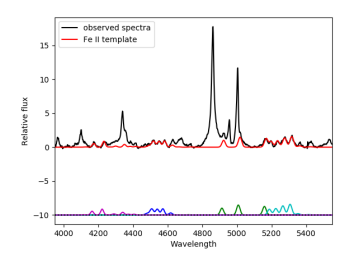

(a) IRAS 03450+0055

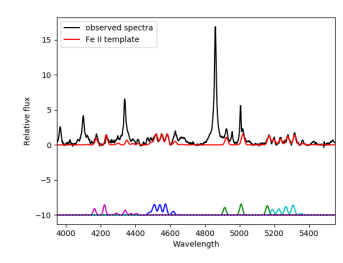

(b) Mkn 1044

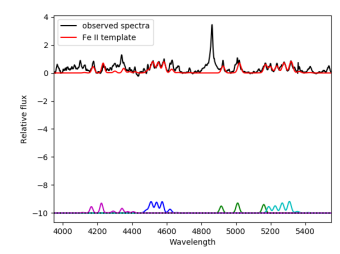

(c) $1 \mathrm{H} 0707-495$

Figure 1: Figures 1a, 1b, and 1c show the Fe II template fitted to the observed spectra.

1H 0707-495 exhibits weaker forbidden lines (eg. [O III]) compared to Mkn 1044 and IRAS 03450+0055. Relative to other emission lines in the spectrum, the Fe II lines of 1H 0707-495 are in turn stronger compared to the other two objects (Figures 1a, 1b, and 1c). These differences indicate that there are possibly some structural differences and therefore different gas dynamics are responsible for the AGN activity in 1H0707-495. The forbidden emission lines are strongest in IRAS $03450+0055$.

No notable long-term flux variations were found between adjacent epochs (Figure 3), including $\mathrm{H} \beta(<10 \%)$ (Figure 3a), and X-rays(<34\% for $1 \mathrm{H} 0707-495$, and $<60 \%$ for Mkn 1044) (Figure $3 e)$. The flux change in Fe II lines for all the three objects is also at best minimal $(<18 \%$, with a 


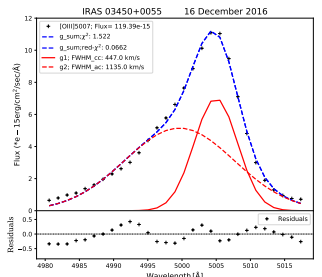

(a)

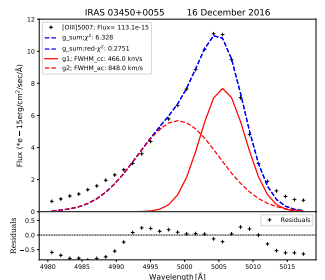

(b)

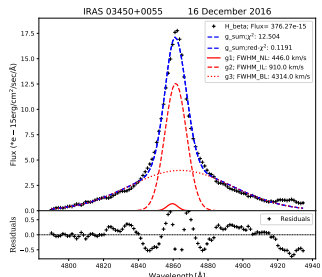

(c)

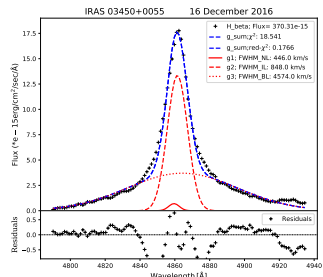

(d)

Figure 2: Comparison of fitting of the [O III] $5007 \AA$ and $\mathrm{H} \beta$ lines of IRAS $03450+0055$. The components of the line [O III] $5007 \AA$ were fitted freely (Figure 2a) while the asymmetric blue wing of the same line was fixed to match the Fe II profile (Figure $2 b$ ). Figures $2 \mathrm{c}$ and $2 \mathrm{~d}$ show the $\mathrm{H} \beta$ line fits with their narrow component set to $10 \%$ of that of [O III] $5007 \AA$ with its same width and shift. In figure $2 \mathrm{~d}$, the intermediate component of $\mathrm{H} \beta$ was set to fit in line with the Fe II profile.

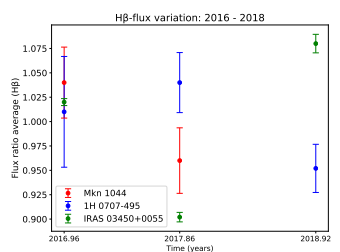

(a)

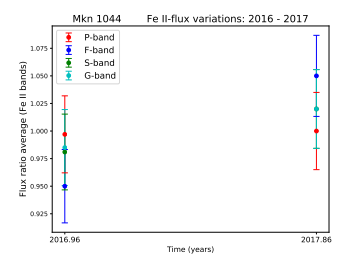

(b)

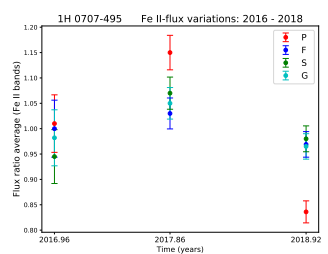

(c)

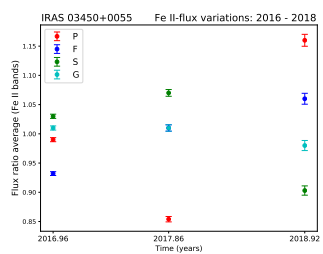

(d)

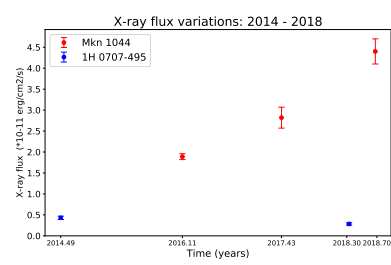

(e)

Figure 3: The figures compare different line flux variations over a certain time period for multiple epochs of the three objects. The X-ray fluxes recorded in Figure $3 \mathrm{e}$ were extracted from the Swift Archive Data for the objects available for the period closest to the corresponding optical observations. All values were plotted with error bars as shown.

majority of Fe II bands varying less than 4\%) as shown in as shown in Figures 3b, 3c, and 3d. By comparison, the X-ray flux variations of Mkn 1044 and 1H 0707-495 are much greater than in the optical (Figure 3e).

For both Mkn 1044 and IRAS 03450+0055, the [O III]5007 $\AA$ line is a combination of a symmetric core component and an asymmetric component (the blue wing). This profile is common in $\operatorname{AGN}[21,22,20]$, and was fitted as the sum of two individual Gaussian functions. The asymmetric component is however not evident in 1H 0707-495. This blue wing of the [O III] line and the ILR 


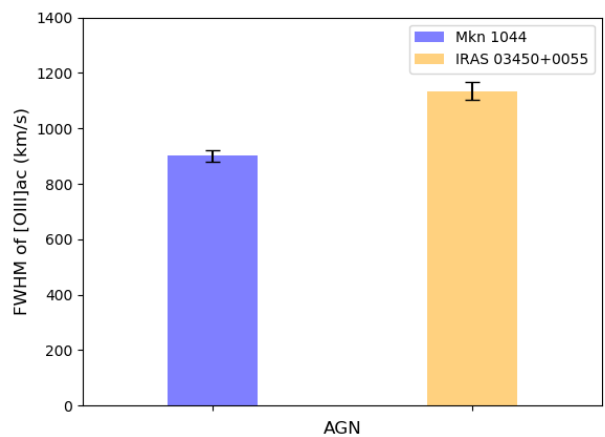

(a)

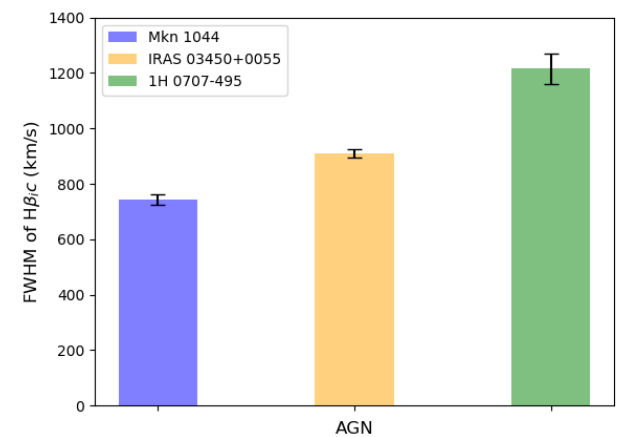

(b)

Figure 4: This figure displays the fitted widths (and standard deviation thereof) of [O III] $] 5007 \AA$ (Figure 4a) and $\mathrm{H} \beta$ (Figure 4b) in line with the Fe II profiles of the AGNs. No asymmetric [O III] component was detected in 1H 0707-495.

component of $\mathrm{H} \beta$ fit the Mkn 1044 and IRAS $03450+0055$ spectra reasonably well with widths similar to those of Fe II (Figures $2 \mathrm{~b}$ and $2 \mathrm{~d}$ ). The majority of epochs indicate a fitting error within the range \pm 50 to $\pm 150 \mathrm{~km} / \mathrm{s}$ or $5.6 \%-18.3 \%$ (Figure $4 \mathrm{a}$ ).

The $\mathrm{H} \beta$ line is fitted as the sum of three Gaussians which corresponds to a narrow line, an intermediate line and a broad line. In general, this component could be fitted satisfactorily with a width similar to that of Fe II, and with an error of less than $20 \mathrm{~km} / \mathrm{s}$ (or $<1.80 \%$ (Figure $4 \mathrm{~b}$ ).

It is suggested that the blue-shifted [O III] $5007 \AA$ line is mainly related to nuclear outflows and strong winds $[21,22,20]$. The shocks produced from AGN can act as a source of excitation which leads to nuclear photoionization. The gas that flows out of the inner part of the NLR might also be affected by the wind from the nucleus that could explain a blue-shifted [O III] line. The widths of $\mathrm{H} \beta$ ILR component and [O III] blue wing reasonably match the corresponding Fe II line width. This suggests that both originate in the same region between the NLR and the BLR with the same gas dynamics of the so called intermediate line region.

\section{Conclusion}

1. Mkn 1044 and IRAS 03450+0055 show extended [O III] blue wings with a line width of around $1000 \mathrm{~km} / \mathrm{s}$, which is in agreement with some of the previous observations of NLS1 galaxies [3, 23, 24, 22].

2. Both [O III] blue wing and $\mathrm{H} \beta$ ILR component are fitted satisfactorily by profiles similar to those of Fe II, suggesting that they all originate in the same region.

3. The blue wing is not very evident in 1H0707-495. This AGN also has comparatively weak forbidden lines. This possibly indicates that some structural differences and different gas dynamics are responsible for the AGN activity in 1H 0707-495 compared to the other two AGN.

4. No dramatic flux variations are found in $\mathrm{H} \beta(<10 \%)$, Fe II bands $(<18 \%$, with the majority of Fe II bands varying less than 4\%), and in X-rays ( $<34 \%$ for $1 \mathrm{H} 0707-495$, and $<60 \%$ for Mkn 1044 
between adjacent epochs).

5. The minimum variability found in Fe II lines suggests that they originate not close to the central engine, i.e. far from the accretion disk.

\section{Acknowledgements}

Bynish Paul acknowledges the receipt of a PhD bursary through the South African Astronomical Observatory from the National Research Foundation of South Africa. We thank Francois van Wyk for occasional assistance at the telescope.

\section{References}

[1] J. Kovačević, L. C. Popović and M. S. Dimitrijević, Analysis of optical Fe II emission in a sample of active galactic nucleus spectra, Astrophys. J. 189 (2010) 15.

[2] T. A. Boroson and R. F. Green, The emission-line properties of low-redshift quasi-stellar objects, Astrophys. J. 80 (1992) 109.

[3] M.-P. Veron-Cetty, P. Veron and A. C. Goncalves, A spectrophotometric atlas of Narrow-Line Seyfert 1 galaxies, Astron. Astrophys. 372 (2001) 730 [astro-ph/0104151].

[4] D. E. Osterbrock and R. W. Pogge, The spectra of narrow-line Seyfert 1 galaxies, Astrophys. J. 297 (1985) 166.

[5] R. W. Goodrich, Spectropolarimetry of 'narrow-line' Seyfert 1 galaxies, Astrophys. J. 342 (1989) 224.

[6] J. E. Steiner, A spectrophotometric classification of low-redshift quasars and active galactic nuclei, Astrophys. J. 250 (1981) 469.

[7] D. Grupe, A complete sample of soft X-ray-selected AGNs. II. Statistical analysis, Astron. J. 127 (2004) 1799.

[8] J. W. Sulentic, P. Marziani, S. Zamfir, Comparing H $\beta$ Line Profiles in the $4 D$ Eigenvector 1, New Astronomy Reviews 53 (2009) 198 [astro-ph.CO].

[9] S. Zamfir, J. W. Sulentic, P. Marziani and D. Dultzin, Detailed characterization of H $\beta$ emission line profile in low-z SDSS quasars, Mon. Not. R. astr. Soc. 403 (2010) 1759.

[10] S. Collin and M. Joly, The Fe II problem in NLS1s, New Astronomy Reviews 44 (2000) 531 [astro-ph/0005153v1].

[11] E. M. Giannuzzo and G. M. Stirpe, A search for variability in Narrow Line Seyfert 1 Galaxies, Astron. Astrophys. 314 (1996) 419.

[12] L. C. Popović, E. Mediavilla, E. Bon and D. Ilić, Contribution of the disk emission to the broad emission lines in AGNs: Two-component model, Astron. Astrophys. 423 (2004) 909. 
[13] C. Hu, J.-M. Wang, L. C. Ho, Y.-M. Chen et al, A Systematic Analysis of Fe II Emission in Quasars: Evidence for Inflow to the Central Black Hole, Astrophys. J. 687 (2008) 78.

[14] M. Gaskell, What broad emission lines tell us about how active galactic nuclei work, New Astronomy Reviews 53 (2009) 140.

[15] S. Collin-Souffrin, S. Dumont, N. Heidmann and M. Joly, Formation of permitted lines in the spectrum of type 1 Seyfert galaxies and quasars. II - Fe II lines and the low excitation region, Astron. Astrophys. 83 (1980) 190.

[16] X.-G. Zhang, D. Dultzin-Hacyan and T.-G. Wang, SDSS J2125-0813: the evidence for the origination of optical Fe II emission lines from an accretion disc near a central black hole, Mon. Not. R. astr. Soc. 372 (2006) L5.

[17] T. Nagao, T. Murayama and Y. Taniguchi, The Narrow-Line Region of Seyfert Galaxies: Narrow-Line Seyfert 1 Galaxies versus Broad-Line Seyfert 1 Galaxies, Astrophys. J. 546 (2001) 744.

[18] M. Joly, An unsolved problem in the spectrum of quasars: the Fe II emission lines, Annales de Physique 18 (1993) 241.

[19] L. C. Popović and J. Kovačević, Optical emission-line properties of a sample of the broad-line active galactic nuclei: the Baldwin effect and eigenvector 1, Astrophys. J. 738 (2011) 68.

[20] E. O. Schmidt, G. A. Oio, D. Ferreiro, L. Vega1 and W. Weidmann, Asymmetric emission of the [O III] 5007 profile in narrow-line Seyfert 1 galaxies, Astron. Astrophys. 615 (2018) A13.

[21] T. A. Boroson and J. B. Oke, Spectroscopy of the galaxy components of $N$ and Seyfert galaxies, Publs. Astron. Soc. Pacific, 99 (1987) 809.

[22] P. Marziani, J. W. Sulentic, R. Zamanov, M. Calvani et al., An Optical Spectroscopic Atlas of Low-Redshift Active Galactic Nuclei, Astrophys. J. Suppl. 145 (2003) 199.

[23] W. Bian, Q. Yuan and Y. Zhao, [O III] emission line in narrow-line Seyfert 1 galaxies, Mon. Not. R. astr. Soc. 367 (2006) 860.

[24] S. Komossa, W. Voges, D. Xu, S. Mathur, H.-M. Adorf et al, Radio-loud narrow-line type 1 quasars, Astrophys. J. 132 (2006) 531. 